The BMJ

Cite this as: $B M J 2021 ; 375: \mathrm{n} 3079$

http://dx.doi.org/10.1136/bmj.n3079

Published: 14 December 2021
NEWS ANALYSIS

\section{Covid-19: Omicron and the need for boosters}

Over 365 million booster or third vaccine doses have been administered globally. But what protection
do they offer against the latest covid variant? Elisabeth Mahase reports

Elisabeth Mahase

Has omicron increased the need for boosters?

Booster doses of covid-19 vaccines have been rolled out in many countries since the summer because of concerns over waning immunity and the risk of another wave of infections over the winter. ${ }^{1}$ However, new concerns about the more transmissible omicron variant and its apparent ability to reduce vaccine effectiveness have led to some countries, including the UK, to speed up their booster rollout in an attempt to keep immunity levels high.

The UK Health Security Agency has said that early estimates indicated that omicron significantly reduced the effectiveness of vaccines against symptomatic infection, when compared with the previously dominant delta infection. But a booster dose leads to a moderate to high vaccine effectiveness of $70 \%$ to $75 \%$. It's still unclear whether omicron will affect the ability of vaccines to protect against hospital admissions and deaths, but the UKHSA said that, on the basis of past experience, this protection "is likely to be substantially higher than the estimates against symptomatic disease."

Much of the attention has focused on what's happening in South Africa, where omicron was first detected. A very small study, released as a preprint and involving just 12 people in South Africa, ${ }^{3}$ indicated that concentrations of neutralising antibodies after the Pfizer vaccine were 41 -fold lower in the presence of omicron than were seen with the variant of SARS-CoV-2 that was widespread in the early stages of the pandemic (characterised by spike protein substitution D614G).

These findings have been supported by the latest report (11 December) from the UK's Com-COV2 study, which found a "substantial fall in neutralisation titres" with the omicron variant in people who had had two doses of the Pfizer or AstraZeneca vaccines. ${ }^{4}$ The authors of this preprint pointed out that some recipients failed to neutralise "at all." They said that these data suggested that omicron was more "antigenically distant from the original SARS-CoV2 vaccine strain than the previously most distant strains beta and delta."

The researchers reported, "This will likely lead to increased breakthrough infections in previously infected or double vaccinated individuals, which could drive a further wave of infection, although there is currently no evidence of increased potential to cause severe disease, hospitalisation or death ... However, it should be noted that higher transmission will inevitably lead to increased numbers of cases and greater burden on health systems, even without proportional changes in severity."

Nonetheless, some commentators have argued that it is unethical to give additional vaccine doses when much of the world's population is yet to receive their first dose. It is estimated that $44 \%$ of people in the world have not had a single dose of a covid vaccine and that only $7.1 \%$ of people in low income countries have received at least one dose. ${ }^{5}$

\section{What effect do booster doses have?}

In September researchers in Israel, one of the earliest countries to roll out boosters, reported that a third dose of the Pfizer vaccine substantially cut rates of infection and of severe illness in people aged over 60 , when compared with those who had only two doses. Using data from more than 1.1 million people aged 60 or over (30 July to 31 August 2021), they found that at least 12 days after the booster dose the rate of confirmed infection was lower in the booster group than in the non-booster group by a factor of 11.3 (95\% confidence interval 10.4 to 12.3). The rate of severe illness was also lower in the booster group, by a factor of 19.5 (12.9 to 29.5$){ }^{6}$

Since then, different covid vaccines have been found to be safe and effective as a booster dose. The Cov-Boost trial looked at use of seven different vaccines as boosters after two doses of either the AstraZeneca or Pfizer vaccines: AstraZeneca, Curevac, Johnson and Johnson (Janssen), Moderna, Novavax, Pfizer, and Valneva. The trial found that all vaccines (except for Curevac, which was withdrawn) boosted the immune response, although the level of antibodies differed significantly, depending on the mix of vaccines. ${ }^{7}$

Among people primed with two doses of AstraZeneca, antibody levels were 32 (99\% confidence interval 24.8 to 42) times higher after the Moderna booster than in the control group, whereas after a half dose of Valneva the levels were 1.8 (1.5 to 2.3) times higher. Among people primed with the Pfizer vaccine, a Moderna booster increased antibody levels 11.5 (9.4 to 14.1) times higher than control, while a half dose of Valneva increased levels by 1.3 times (1.0 to 1.5). The results were similar in all age groups.

Separately, Pfizer has reported that a third dose of its vaccine provided levels of neutralising antibodies against omicron that were similar to those seen after two doses of the vaccine against the original virus (wildtype).

Mahase E. Covid-19 booster vaccines: What we know and who's doing what. BMJ2021;374:n2082. doi: 10.1136/bmj.n2082 pmid: 34417167 
2 UK Health Security Agency. SARS-CoV-2 variants of concern and variants under investigation in England. Dec 2021. https://assets.publishing.service.gov.uk/government/uploads/system/uploads/attachment_data/file/1040076/Technical_Briefing_31.pdf.

3 Mahase E. Covid-19: Do vaccines work against omicron-and other questions answered. BMJ 2021;375:n3062. doi: 10.1136/bmj.n3062 pmid: 34893476

4 Dejnirattisai W, Shaw RH, Supasa P, et al. Reduced neutralisation of SARS-Cov-2 omicron-B.1.1.529 variant by post-immunisation serum. MedRxiv 2021:21267534 [preprint]. https://www.medrxiv.org/content/10.1101/2021.12.10.21267534v1.full.pdf.

5 Our World in Data. Coronavirus (covid-19) vaccinations. Dec 2021. https://ourworldindata.org/covid-vaccinations.

6 Mahase E. Covid-19: Booster dose reduces infections and severe illness in over 60s, Israeli study reports. BMJ 2021;374:n2297. doi: 10.1136/bmj.n2297 pmid: 34535470

7 Mahase E. Covid-19: Antibody boost after third dose varies greatly by vaccine, study finds. BMJ 2021;375:n3011. doi: 10.1136/bmj.n3011 pmid: 34862175

This article is made freely available for use in accordance with BMI's website terms and conditions for the duration of the covid-19 pandemic or until otherwise determined by BMJ. You may use, download and print the article for any lawful, non-commercial purpose (including text and data mining) provided that all copyright notices and trade marks are retained. 